\title{
Antibacterial Membrane with a Bone-Like Structure for Guided Bone Regeneration
}

\author{
YuYuan Zhang, ${ }^{1}$ WeiJia Mao, ${ }^{1}$ Jian Wang, ${ }^{1}$ Quan-Li Li, ${ }^{1}$ May Lei Mei, ${ }^{2}$ \\ Chun Hung $\mathrm{Chu}^{2}{ }^{2}$ Rong $\mathrm{Xia}^{3}{ }^{3}$ and Zhi-Hong Zhang ${ }^{4}$ \\ ${ }^{1}$ College \& Hospital of Stomatology, Anhui Medical University, Key Laboratory of Oral Diseases Research of Anhui Province, \\ No. 69, Meishan Road, Hefei 230000, China \\ ${ }^{2}$ Faculty of Dentistry, The University of Hong Kong, Pokfulam 999077, Hong Kong \\ ${ }^{3}$ Department of Stomatology, The Second Affiliated Hospital of Anhui Medical University, Hefei 230000, China \\ ${ }^{4}$ Department of Stomatology, The Hospital of Anhui Province, Hefei 230000, China
}

Correspondence should be addressed to Quan-Li Li; ql-li@126.com

Received 6 June 2015; Revised 2 September 2015; Accepted 3 September 2015

Academic Editor: Victor M. Castaño

Copyright (C) 2015 YuYuan Zhang et al. This is an open access article distributed under the Creative Commons Attribution License, which permits unrestricted use, distribution, and reproduction in any medium, provided the original work is properly cited.

An antibacterial membrane with a bone-like structure was developed for guided bone regeneration (GBR) by mineralising acellular bovine pericardium (ABP) and loading it with the antibiotic minocycline. The bovine pericardium (BP) membrane was processed using physical and chemical methods to remove the cellular components and obtain ABP membranes. Then, the ABP membranes were biomimetically mineralised using a calcium phosphate-loaded agarose hydrogel system aided by electrophoresis. Minocycline was adsorbed to the mineralised ABP membrane, and the release profile in vitro was studied. The membranes were characterised through scanning electron microscopy, diffuse reflectance-Fourier transform infrared spectroscopy, and X-ray diffraction. Results showed that the ABP membrane had an asymmetric structure with a layer of densely arranged and irregularly aligned collagen fibrils. Collagen fibrils were calcified with the formation of intrafibrillar and interfibrillar hydroxyapatites similar to the bone structure. Minocycline was incorporated into the mineralised collagen membrane and could be released in vitro. This process endowed the membrane with an antibacterial property. This novel composite membrane offers promising applications in bioactive GBR.

\section{Introduction}

Guided bone regeneration (GBR) is a well-established therapy that promotes bone regeneration. GBR is based on a barrier membrane that prevents fibrous and epithelial tissues from invading the bone defect zone and thus blocks osteogenesis [1]. Improving the membrane biocompatibility to induce osteogenesis and using a mechanism to support the space of bone regeneration and antibacterial properties are required for GBR. Bioabsorbable collagen membranes have been successfully used in clinical applications. However, these membranes still present certain limitations, such as inferior mechanical properties and rapid degradation of membranes, which are associated with connective tissue invasion and infection, among others.
Acellular bovine pericardium (ABP) is a typical natural collagen membrane that has attracted considerable interest because of its potential application in bioprosthetics [2-6]. $\mathrm{ABP}$ has also been utilised as a barrier membrane in GBR [7]. $\mathrm{ABP}$ is composed of native extracellular matrices that contain numerous collagen fibres, several elastin fibres, and various glycosaminoglycans with a highly interconnected porous structure (average pore size of $25 \mu \mathrm{m}$ and interconnectivity/porosity of $60 \%$ ) on both the surface and the internal structure.

Bone is a composite material with a basic microstructure characterised by calcified collagen fibrils forming a scaffold as a template for hydroxyapatite (HA) crystal nucleation and growth. Plate-like HA is preferentially oriented with the $c$-axis parallel to the longitudinal axis of the fibrils [8]. 
In our previous study, we have developed a technique to remineralise the dentin collagen matrix [9].

Infection is a significant health care burden and is considered as the major reason for GBR failure in clinical practice. Therefore, antibiotic drug-loaded membranes are highly demanded. However, the incorporation of antibiotics into biomaterials often results in biocompatibility attenuation. Minocycline is a second-generation semisynthetic tetracycline antibiotic that exhibits pleiotropic biologic activities besides its anti-inflammatory activities. Minocycline can also enhance bone formation by inhibiting the activity of osteoclasts, promoting the activity of osteoblasts, and inhibiting connective tissue breakdown and bone resorption [10]. Thus, minocycline is a good candidate for GBR membrane design. We have previously developed a minocycline-releasing HAgelatin hybrid material for bone defect repair [11].

The current study aims to develop an antibacterial membrane with a bone-like structure for GBR. The mechanism, biocompatibility, and antibacterial property of the ABP membrane were improved through mineralisation and minocycline loading.

\section{Materials and Methods}

2.1. BP Source. Fresh BP was obtained from a bovine heart (20-22 months old). External fat and adherences were removed, and BP samples were obtained. The obtained BP samples were stored at $-80^{\circ} \mathrm{C}$ until use in the laboratory.

2.2. BP Decellularisation. The BP samples were freezethawed $\left(-80^{\circ} \mathrm{C}\right.$ for $4 \mathrm{~h}$ followed by $37^{\circ} \mathrm{C}$ for $\left.30 \mathrm{~min}\right)$ for five times and ultrasonically cleansed for $3 \mathrm{~min}$ after each cycle. The samples were immersed in sterile phosphatebuffered saline (PBS) on a shaker $\left(300 \mathrm{rpm} ; 4^{\circ} \mathrm{C}, 24 \mathrm{~h}\right)$, and the medium was refreshed every $6 \mathrm{~h}$. Then, the samples were treated with $0.4 \%$ TritonX-100 for $48 \mathrm{~h}$ on a shaker (300 rpm; $4^{\circ} \mathrm{C}$; the medium was refreshed every $24 \mathrm{~h}$ ) and ultrasonically cleansed for $2 \mathrm{~min}$ after each cycle. The samples were again immersed in PBS on a shaker $\left(300 \mathrm{rpm} ; 4^{\circ} \mathrm{C}\right.$, $24 \mathrm{~h}$; the medium was refreshed every $6 \mathrm{~h}$ ) to obtain the ABP. The acquired ABP was characterised via scanning electron microscopy (SEM) (Sirion 200, FEI Co., Hillsboro, Oregon, USA). For SEM observation, fresh ABP was dehydrated in a graded ethanol series $(50 \%, 70 \%, 80 \%, 90 \%, 95 \%$, and $100 \%)$ and then critical point-dried (Critical Point Drying, K850, Quorum, England) before gold sputtering.

2.3. Agarose Gel Preparation. An agarose gel with $0.13 \mathrm{M}$ $\mathrm{CaCl}_{2}$ was prepared by adding agarose powder $(1.0 \mathrm{~g})$ to $100 \mathrm{~mL}$ of $0.13 \mathrm{M} \mathrm{CaCl}_{2}$ solution. An agarose gel with $0.26 \mathrm{M}$ $\mathrm{Na}_{2} \mathrm{HPO}_{4}$ was prepared by adding agarose powder $(1.0 \mathrm{~g})$ to $100 \mathrm{~mL}$ of $0.26 \mathrm{M} \mathrm{Na}_{2} \mathrm{HPO}_{4}$ solution containing $500 \mathrm{ppm}$ fluoride; $0.1 \mathrm{M} \mathrm{NaOH}$ and $0.1 \mathrm{M} \mathrm{HCl}$ were used to adjust the $\mathrm{pH}$ to 6.5. The mixtures were enhanced for $30 \mathrm{~min}$ and then heated to $100^{\circ} \mathrm{C}$ until the agarose was completely dissolved.

2.4. Mineralisation of ABP Membranes. ABP membranes were mineralised using a calcium phosphate-loaded agarose gel system aided by electrophoresis in accordance with our

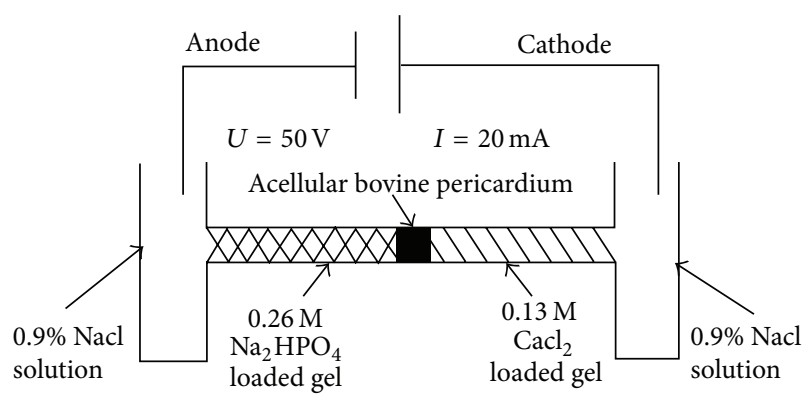

FIGURE 1: Schematic of the electrophoresis-aided mineralising system.

previous protocol [10]. The electrophoresis aiding the mineralising system (Figure 1) consisted of a two-way horizontal polyether tube, two plastic cells, two graphite electrodes, and electrophoresis. Agarose gel with $20 \mathrm{~mL}$ of $0.13 \mathrm{M} \mathrm{CaCl}_{2}$ and $20 \mathrm{~mL}$ of $0.26 \mathrm{M} \mathrm{Na}_{2} \mathrm{HPO}_{4}$ containing $500 \mathrm{ppm}$ fluoride ( $\mathrm{pH}$ 6.5) was placed on the two sides of the tube separated by the ABP membrane. The tube was then connected to the plastic cells. Electrodes were placed at the bottom of the cells, which were filled with $100 \mathrm{~mL}$ of $0.9 \% \mathrm{NaCl}$ solution. The electric current was constantly maintained at $20 \mathrm{~mA}$ during electrophoresis. The gel and $\mathrm{NaCl}$ solution were refreshed every $2 \mathrm{~h}$, and their exchange defined the completion of a mineralisation cycle. ABP was ultrasonically cleansed with $100 \mathrm{~mL}$ of sterile PBS solution for $2 \mathrm{~min}$ after each cycle. The sample mineralised through three, six, and nine cycles was assessed and characterised using SEM, X-ray diffraction (XRD; X' Pert Pro, Philips Almelo, Netherlands), diffuse reflectance-Fourier transform infrared spectroscopy (DRFTIR; Nicolet 8700 Thermo Scientific Instrument Co., Friars Drive Hudson, New Hampshire, USA), thermogravimetric analysis (TGA), and differential thermal analysis (DTA; ATA 449C Jupiter, NETZSCH, Germany).

2.5. Preparation of Minocycline-Loaded Mineralised ABP. Minocycline hydrochloride was dissolved in sterile PBS to obtain a stock solution of $500 \mathrm{mg} / \mathrm{L}$. The mineralised membranes were immersed in $500 \mathrm{mg} / \mathrm{L}$ minocycline solution in a constant-temperature air bath oscillator $(100 \mathrm{r} / \mathrm{min})$ at $37^{\circ} \mathrm{C}$ for $48 \mathrm{~h}$. The membranes were rinsed thrice, ultrasonically cleansed with deionised water for $5 \mathrm{~min}$, and then lyophilised to obtain the final membranes.

The release of minocycline from the composite membranes in vitro was determined by incubating the $10 \mathrm{~mm}$ diameter membranes in $1 \mathrm{~mL}$ of sterile PBS and in a constanttemperature air bath oscillator $(100 \mathrm{r} / \mathrm{min})$ at $37^{\circ} \mathrm{C}$. Individual samples were centrifuged at appropriate time intervals $(1,3$, 5 , and 7 days). The supernatant was collected to measure the content of minocycline, and the same amount of fresh PBS was refilled to continue the test. The amount of minocycline in the supernatant was measured via ultraviolet spectrophotometry at $348 \mathrm{~nm}(n=3)$. The mineralised ABP membrane without minocycline loading treated as above served as the control.

Statistical differences were analysed using ANOVA and deemed significant at $P<0.05$. 


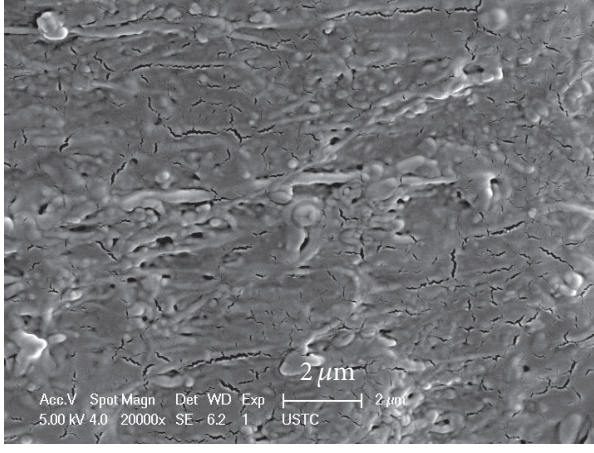

(a)

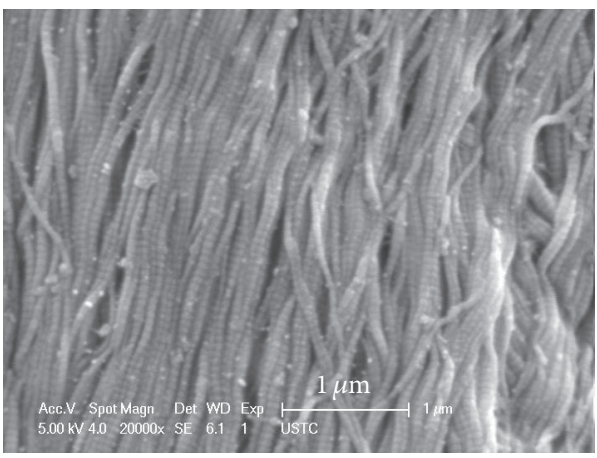

(c)

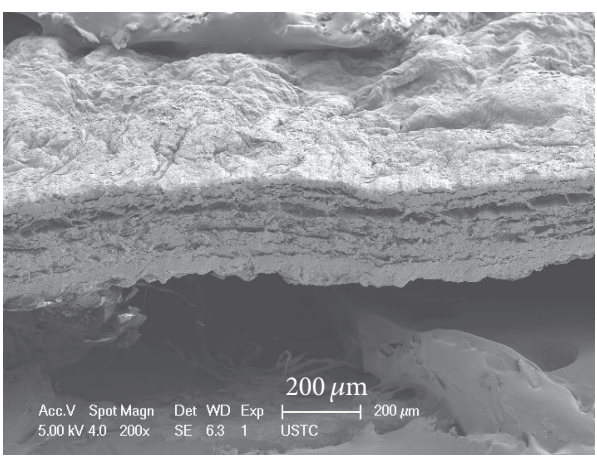

(e)

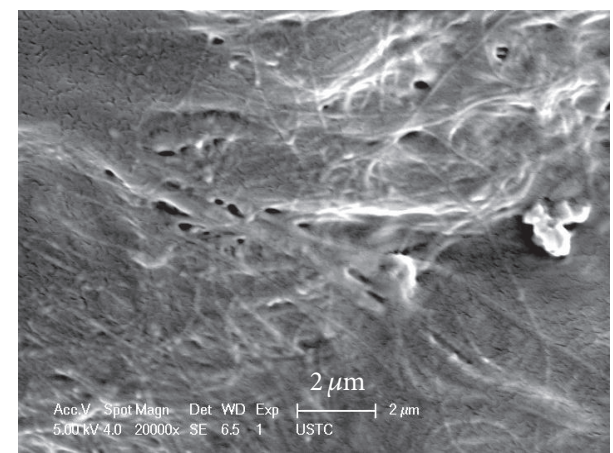

(b)

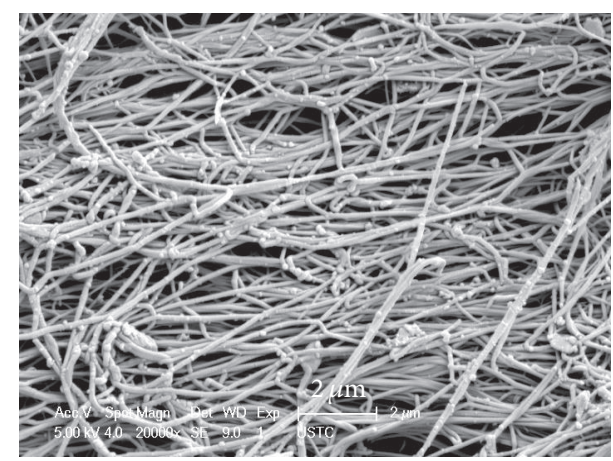

(d)

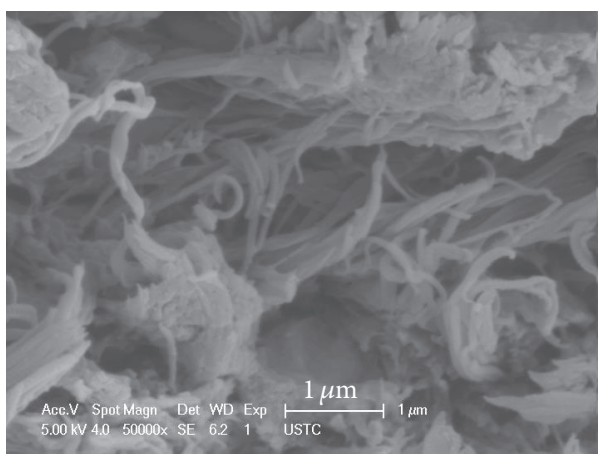

(f)

Figure 2: SEM images of fresh BP membrane ((a) and (b)) and ABP membrane ((c)-(f)). ((a) and (c)) Dense serosal layer surface, ((b) and (d)) loosely and irregularly arranged external layer surface, ((e) and (f)) cross section, and (f) magnification of (e).

\section{Results and Discussion}

3.1. ABP Membrane. Cellular components were removed, and a fibrous matrix with high interconnectivity remained after the decellularisation of fresh BP membrane (Figures 2(a)-2(d)). The ABP membrane showed an asymmetric structure, in which the serosal layer fibres consisted of densely arranged collagen fibrils (Figure 2(c)), whereas the external layer fibres consisted of irregularly aligned collagen fibrils (Figure 2(d)). Given its asymmetric structure, ABP is a highly suitable GBR membrane. The dense layer can effectively isolate the bone defect from the invasion of surrounding connective fibrous tissues, whereas the loose layer can improve adhesion force to bone and stabilise blood clots. By contrast, the ABP membrane possessed a highly interconnected porous structure both on the surface and in its internal structure. Porosity is an important characteristic that enables a barrier membrane to allow nutritional elements to diffuse.

3.2. Mineralised ABP Membrane. The mineralised ABP membrane appeared white. The membrane became relatively stiff, whereas the ABP membrane became soft. Mineralisation also improved surface hardness.

XRD spectra confirmed that the precipitates that formed on the remineralised $\mathrm{ABP}$ membrane were HA crystals (Figure 3). Prior to mineralisation, the ABP membrane showed a typical characteristic of collagen, with wide peaks at approximately $2 \theta=20^{\circ}$ (Figure $3(\mathrm{a})$ ). However, the XRD pattern of the mineralised ABP membranes corresponded 


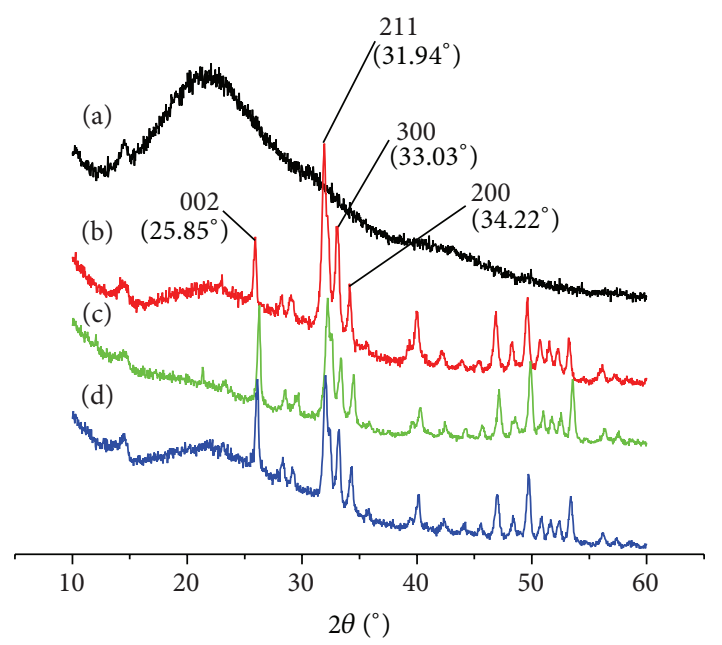

FIGURE 3: XRD spectra of the ABP membrane and the mineralised ABP membrane. (a) ABP membrane; ABP membranes mineralised for (b) three, (c) six, and (d) nine cycles.

well to the expected peaks of HA, such as typical diffraction peaks (002) at $2 \theta=25.8^{\circ},(211)$ at $2 \theta=31.9^{\circ},(112)$ at $2 \theta=32.4^{\circ}$, and (300) at $2 \theta=33.3^{\circ}$. The result of XRD spectra indicated that the ratio of the diffraction intensity of the $c$-axis (002) reflection of HA to the diffraction intensity of the $a$-axis (300) reflection of HA considerably increased as the mineralisation time was prolonged (Figure $3(\mathrm{~b}-\mathrm{d})$ ). This finding suggested that HA precipitates were located along the $c$-axis on the ABP membrane surface and corresponded well to the SEM observation (Figure 4(c)).

In the FTIR spectrum of the ABP membrane (Figure 4(a)), the peak at $3616 \mathrm{~cm}^{-1}$ was ascribed to $\mathrm{NH}_{3}$ groups ( $\mathrm{N}-\mathrm{H}$ vibrational bands); the peaks at 1631,1553 , and $3616 \mathrm{~cm}^{-1}$ were ascribed to amide I $(\mathrm{C}=\mathrm{O}$ vibrational band), amide II (N-H bending band), and amide III (C-N vibrational band), respectively; and the peaks at 1246 and $1077 \mathrm{~cm}^{-1}$ were ascribed to $-\mathrm{OH}$ groups. These peaks exhibit the character of collagen protein. The FTIR spectra of the mineralised $A B P$ membranes were characterised by the predominant distinctive $v_{1} \mathrm{PO}_{4}$ vibrational bands (P-O) at approximately $1023 \mathrm{~cm}^{-1}$ (Figure $4(\mathrm{~b}-\mathrm{d})$ ). In the mineralised ABP membrane, the peaks ascribed to amide groups gradually diminished when the number of mineralising cycles was increased.

The SEM images in Figure 5 show that the collagen fibrils of the ABP membranes were completely mineralised from the surface to the interior. As the mineralisation time was prolonged, many HA crystals formed on the ABP membranes, and the spaces between the collagen fibrils were occupied by HA crystals. After $6 \mathrm{~h}$ of mineralisation (three mineralising cycles), nano-HA crystals nucleated and increased along the collagen fibrils, resulting in their calcification (Figures 5(a), $5(\mathrm{~d})$, and $5(\mathrm{~g}))$. However, the internal cross-sectional images show that several collagen fibrils were not calcified. This result indicated that calcification was slower in the internal structure than on the surface. The number of HA crystals that formed on the ABP collagen scaffolds increased after

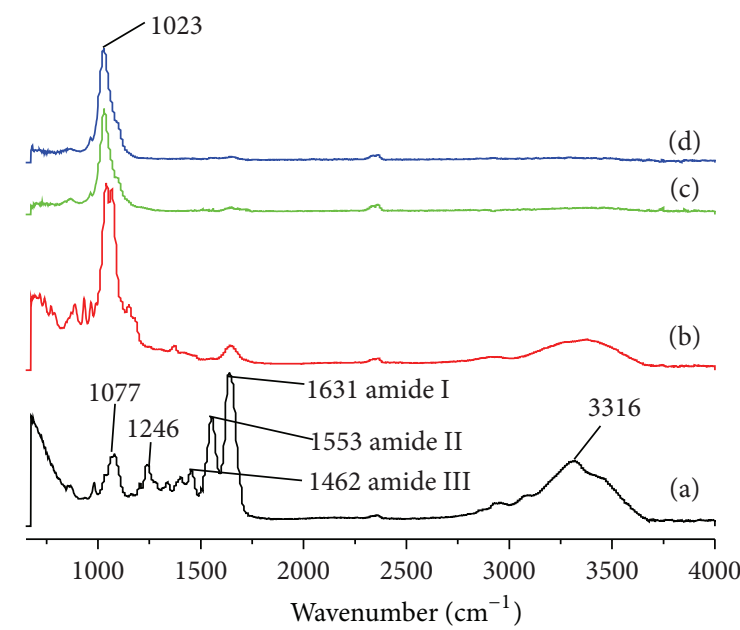

FIGURE 4: DR-FTIR spectra of the ABP membrane and the mineralised $\mathrm{ABP}$ membrane. (a) $\mathrm{ABP}$ membrane; $\mathrm{ABP}$ membranes mineralised for (b) three, (c) six, and (d) nine cycles.

$12 \mathrm{~h}$ of mineralisation (six mineralising cycles) (Figures 5(b), $5(\mathrm{e})$, and $5(\mathrm{~h})$ ). Moreover, the dense serosal layer surface was completely covered by HA crystal clusters (Figure 5(b)). After $18 \mathrm{~h}$ of mineralisation (nine mineralising cycles), collagen fibrils were hardly identified, and densely packed needleshaped HA crystals were arranged perpendicular to the dense serosal layer surface with its $c$-axis (Figure 5(c)).

The basic microstructure of bone comprises a calcified collagen matrix, in which the apatite phase is classified into intrafibrillar and interfibrillar crystallites. Intrafibrillar crystallites occupy interstitial spaces (e.g., hole zones and pore spaces) that separate collagen fibrils [9]. The SEM images in Figure 6 show that precipitated particles were regularly and homogenously distributed along the collagen fibrils in a "string-of-beads" structure on the ABP membrane surface and in the interior, indicating intrafibrillar mineralisation. With substantial mineralisation, intrafibrillar minerals may act as apatite seed crystallites to facilitate the growth of nanocrystals along the collagen fibril, resulting in continued mineral buildup on the exterior and the formation of interfibrillar mineralisation. Interfibrillar HA was embedded between collagen fibrils, exhibiting a "corn-on-the-cob" appearance in certain regions (Figure 6) because of interfibrillar mineralisation. These observations may correspond well with interfibrillar or extrafibrillar mineralisation, which shows the natural appearance of a bone structure. Thus, we confirmed that the mineralisation can create a bone-like structure.

The TGA/DTA data for ABP and mineralised (12 mineralised cycles) membranes are shown in Figure 7. The TGA curve illustrates the mass of ABP samples at different temperatures. The weight of the ABP membrane gradually decreased by $12.916 \%$ from $32.47^{\circ} \mathrm{C}$ to $168.41^{\circ} \mathrm{C}$ and that of the mineralised $\mathrm{ABP}$ membrane gradually decreased by $4.695 \%$ from $24.2^{\circ} \mathrm{C}$ to $103.7^{\circ} \mathrm{C}$. This decrease corresponded to the loss of adsorbed water, which was indicated by a small endothermic peak in the DTA curves. The weight of the 


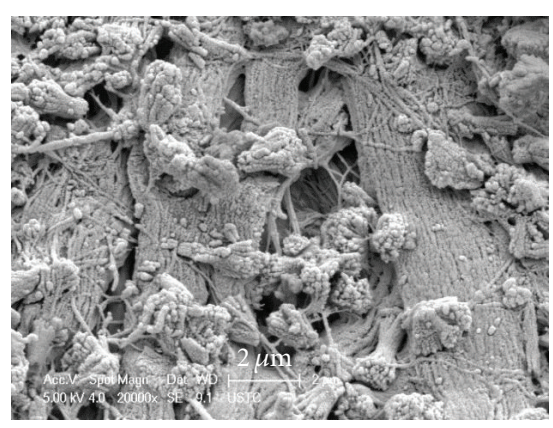

(a)

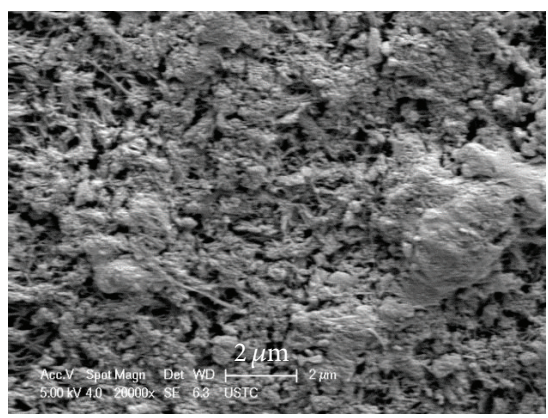

(d)

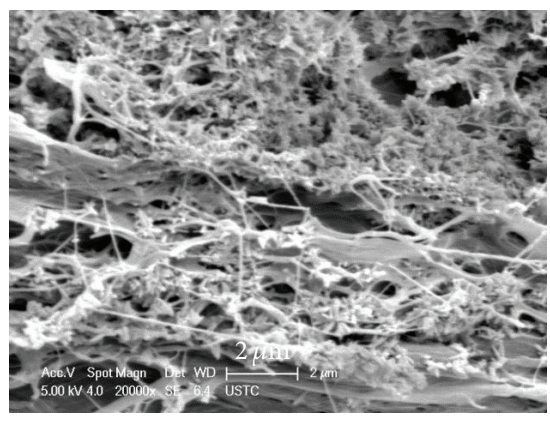

(g)

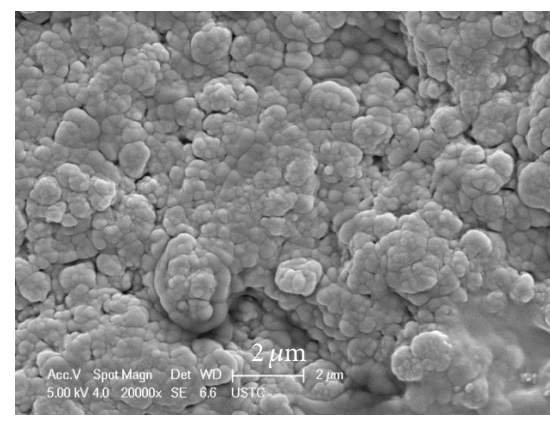

(b)

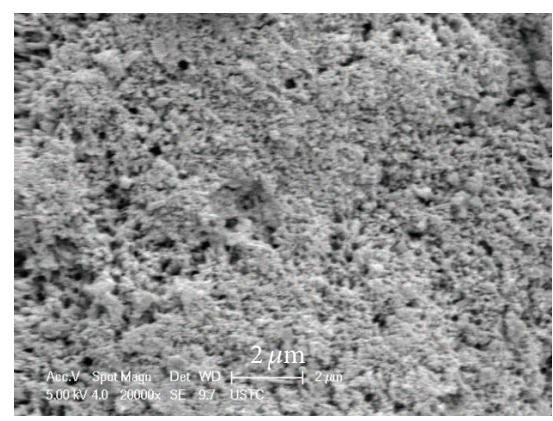

(e)

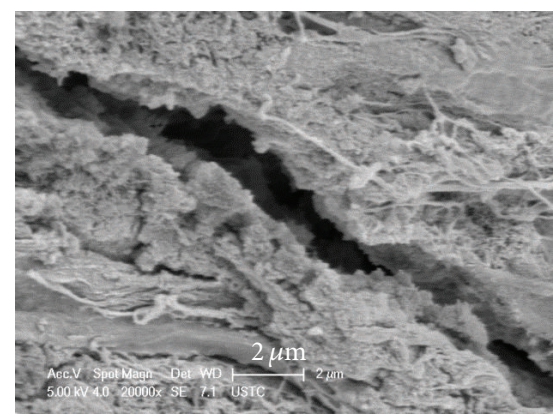

(h)

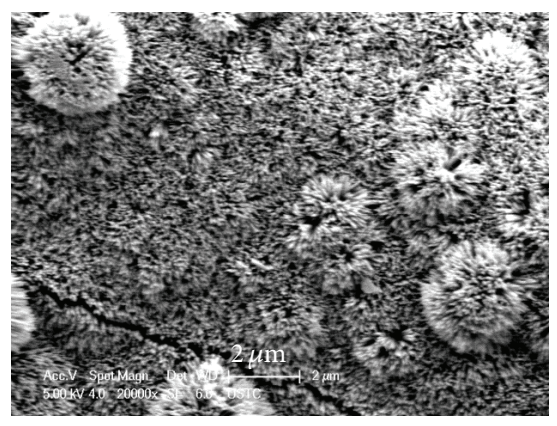

(c)

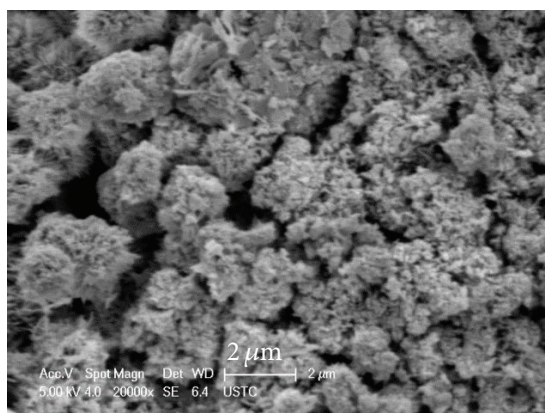

(f)

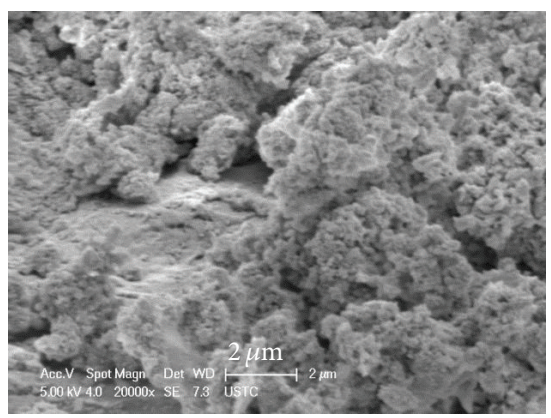

(i)

FIGURE 5: SEM images of the ABP membrane mineralised for three ((a), (d), and (g)), six ((b), (e), and (h)), and nine ((c), (f), and (i)) cycles. ((a), (b), and (c)) Serosal layer surface; ((d), (e), and (f)) external layer surface; and ((g), (h), and (i)) internal cross section.

ABP membrane sharply decreased by $53.588 \%$ from $168.41^{\circ} \mathrm{C}$ to $638.38^{\circ} \mathrm{C}$ and that of the mineralised ABP membrane by only $25.570 \%$ from $203.7^{\circ} \mathrm{C}$ to $561.7^{\circ} \mathrm{C}$. These changes may be attributed to organic collagen decomposition, which was indicated by a small endothermic peak in the DTA curves. The overall weight loss of the ABP sample was $76.504 \%$ (i.e., $23.496 \%$ remained), whereas that of the remineralised ABP sample was $30.265 \%$ (i.e., $69.735 \%$ remained). Thus, the mineralised inorganic component (HA crystals) should be approximately $46.239 \%$ (the reminding remineralised ABP sample $69.735 \%$ minus the remaining organic component $23.496 \%$, namely, $69.735 \%$ minus $23.496 \%$ ). The inorganic mineral component had a structure similar to that of the bone for the mineralised ABP membrane after $18 \mathrm{~h}$ of remineralisation. This result confirms the successful establishment of a bone-like structure.

Many researchers have developed several polymerinorganic composite membranes to mimic the bone hybrid structure; these composite membranes include poly $(\mathrm{L}-$ lactide)/poly(lactic-co-glycolic acid) [12], poly(L-lactideco-glycolide-co- $\varepsilon$-caprolactone) [13], polycaprolactone [14], poly(L-lactic acid)-polysiloxane [15], chitosan-silica [16], and poly(L-lactide-co- $\varepsilon$-caprolactone) [17]. These composite membranes exhibit improved mechanical strength and biocompatibility. However, composite materials are composed of inorganics and organics that are generally not collagen, thereby creating a bone structure that considerably differs from the natural bone structure. Compared with these composite materials, the mineralised ABP membrane is more compatible because of its capacity to support the formation of a bone-like structure.

3.3. Mineralised ABP Membrane Loading Minocycline and Its Release Profile. After minocycline loading, the membrane turned faint yellow, whereas the mineralised ABP membrane without minocycline became white. Figure 8 illustrates the 


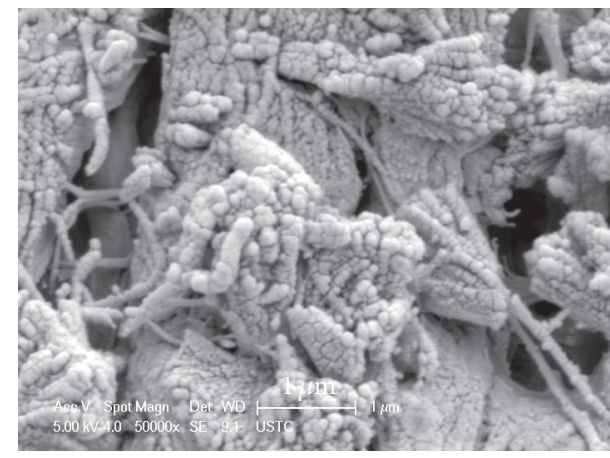

(a)

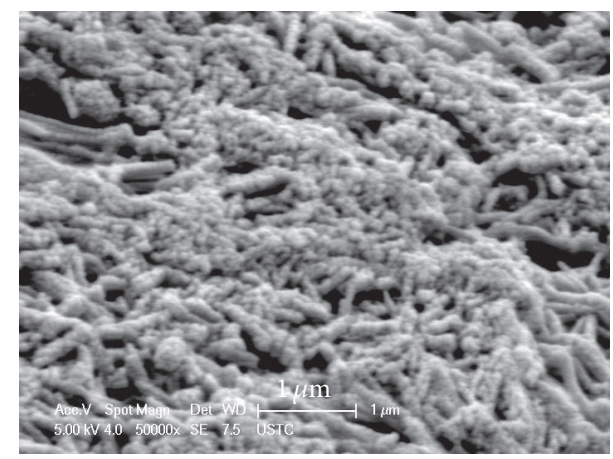

(b)

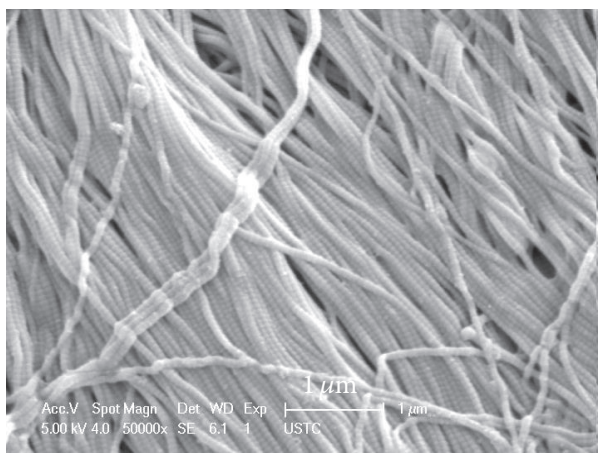

(c)

FIGURE 6: SEM images of the mineralised ABP membrane featuring (a) intrafibrillar mineralisation and (b) interfibrillar mineralisation and the unmineralised ABP membrane (c).

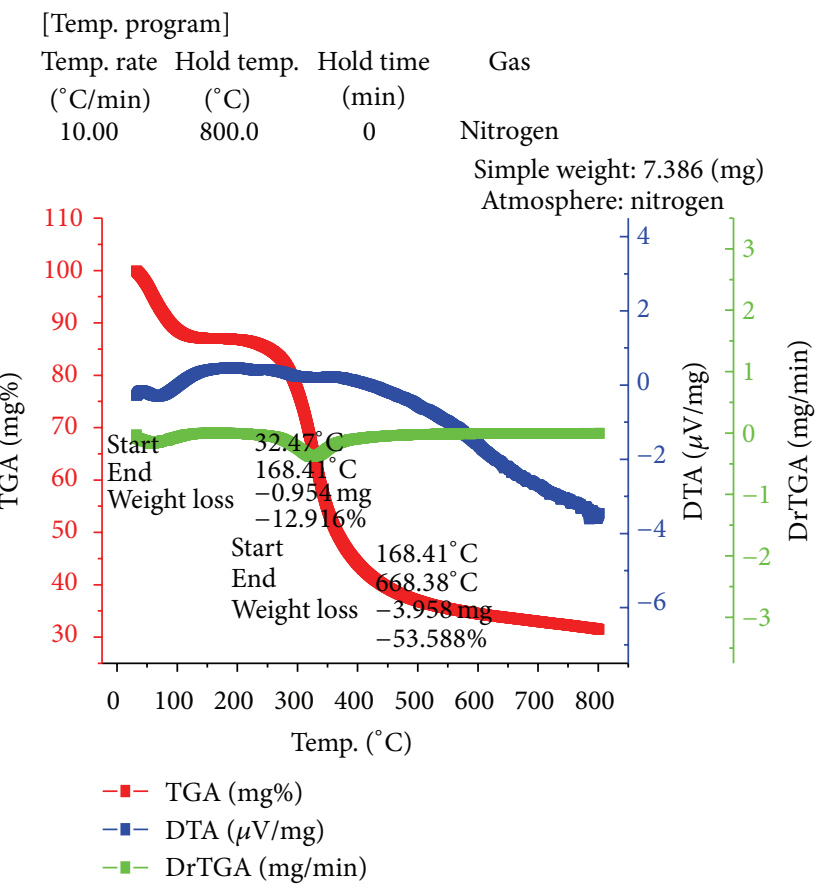

(a)

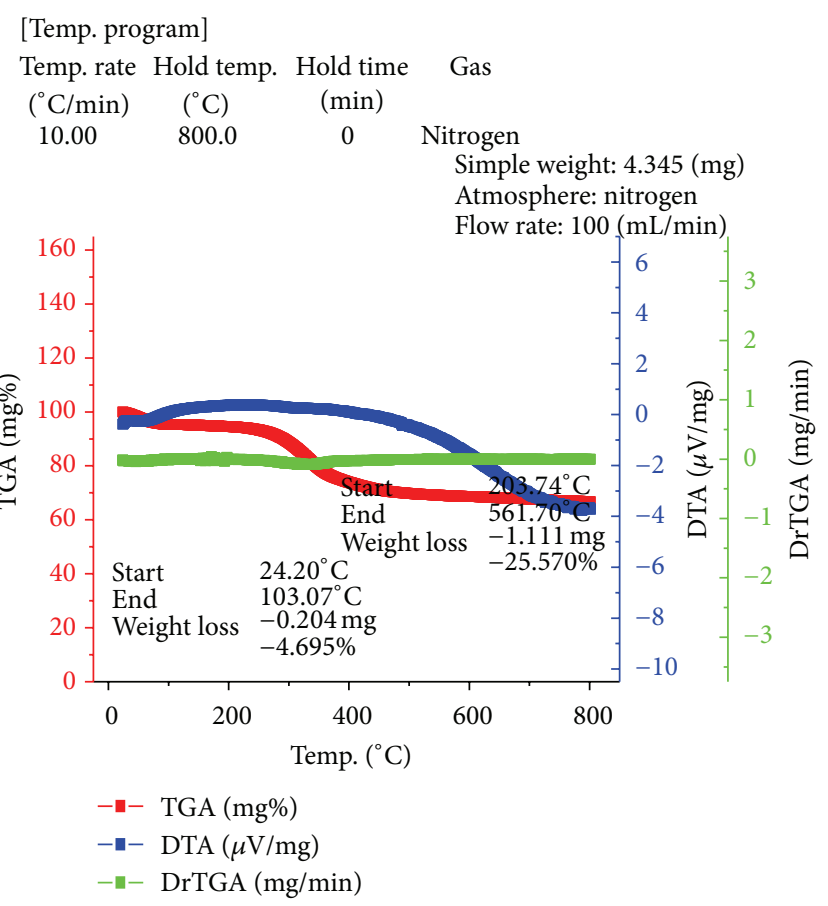

(b)

FIgURE 7: Thermogravimetric and differential thermal analysis data of the (a) ABP membrane and (b) ABP membrane mineralised for $18 \mathrm{~h}$. 


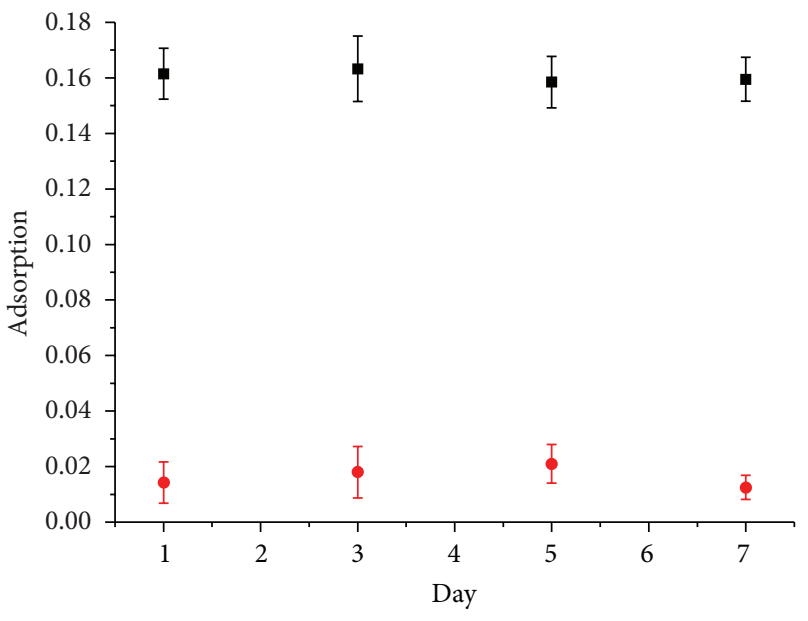

- Mineralised ABP membrane with minocycline loading - Mineralised ABP membrane without minocycline loading

FIGURE 8: Minocycline release curve of mineralised ABP membrane loaded with and without minocycline in vitro.

time-dependent release of minocycline from the composite membrane into the supernatant. No significant difference in the amount of released minocycline $(P>0.05)$ was observed, suggesting that the minocycline release remained nearly constant. Minocycline is a semisynthetic derivative of tetracycline. This compound can bind to HA crystals, and this binding property may contribute to its constant release. The nature of its release differs from that observed in our previous study [11] possibly because the extremely small amount of minocycline cannot overcome the binding ability to HA crystals. In other words, minocycline is bound under saturation, and only a small amount of passive adsorbed minocycline can be found on the composite.

As discussed above, we developed a novel low-cost GBR membrane with a bone-like structure and a minocyclinereleasing property by using simple devices and basic materials. This membrane may improve the mechanical and antibacterial properties and biocompatibility of the collagen membrane. Further, the current membrane can be improved by loading with bioactive molecules, such as growth factors $[18,19]$. Certainly, further biological evaluation is needed to substantiate this claim.

\section{Conclusion}

We developed an antibacterial GBR membrane with a bonelike structure composed of a calcified collagen matrix and minocycline. This composite GBR membrane may provide a potential base to develop other bioactive GBR membranes.

\section{Conflict of Interests}

The authors declare that there is no conflict of interests regarding the publication of this paper.

\section{Acknowledgments}

This work was supported by Grant no. 81061160511 and Grant N_HKU 776/10 through the National Natural Science Foundation of China/Research Grants Council Joint Research Scheme and by Grant no. 9021091203 through key programs for science and technology development of Anhui province.

\section{References}

[1] R. Dimitriou, G. I. Mataliotakis, G. M. Calori, and P. V. Giannoudis, "The role of barrier membranes for guided bone regeneration and restoration of large bone defects: current experimental and clinical evidence," BMC Medicine, vol. 10, article 81, 2012.

[2] G. Goissis, A. de Fátima Giglioti, and D. M. Braile, "Preparation and characterization of an acellular bovine pericardium intended for manufacture of valve bioprostheses," Artificial Organs, vol. 35, no. 5, pp. 484-489, 2011.

[3] A. Gubitosi, G. Docimo, D. Parmeggiani et al., "Acellular bovine pericardium dermal matrix in immediate breast reconstruction after Skin Sparing Mastectomy," International Journal of Surgery, vol. 12, supplement 1, pp. 205-208, 2014.

[4] J. Shen and Z. W. Xu, "Combined application of acellular bovine pericardium and hyaluronic acid in prevention of postoperative pericardial adhesion," Artificial Organs, vol. 38, no. 3, pp. 224230, 2014.

[5] S. Mathapati, D. K. Bishi, S. Guhathakurta et al., "Biomimetic acellular detoxified glutaraldehyde cross-linked bovine pericardium for tissue engineering," Materials Science and Engineering $C$, vol. 33, no. 3, pp. 1561-1572, 2013.

[6] X. C. Dong, X. F. Wei, W. Yi et al., "RGD-modified acellular bovine pericardium as a bioprosthetic scaffold for tissue engineering," Journal of Materials Science: Materials in Medicine, vol. 20, no. 11, pp. 2327-2336, 2009.

[7] M. Bai, T. Zhang, T. Ling et al., "Guided bone regeneration using acellular bovine pericardium in a rabbit mandibular model: invitro and in-vivo studies," Journal of Periodontal Research, vol. 49, no. 4, pp. 499-507, 2014.

[8] G. M. Luz and J. F. Mano, "Mineralized structures in nature: examples and inspirations for the design of new composite materials and biomaterials," Composites Science and Technology, vol. 70, no. 13, pp. 1777-1788, 2010.

[9] X.-T. Wu, Y. Cao, M. L. Mei, J.-L. Chen, Q.-L. Li, and C. H. $\mathrm{Chu}$, "An electrophoresis-aided biomineralization system for regenerating dentin- and enamel-like microstructures for the self-healing of tooth defects," Crystal Growth \& Design, vol. 14, no. 11, pp. 5537-5548, 2014.

[10] M. O. Griffin, G. Ceballos, and F. J. Villarreal, “Tetracycline compounds with non-antimicrobial organ protective properties: possible mechanisms of action," Pharmacological Research, vol. 63, no. 2, pp. 102-107, 2011.

[11] X.-C. Dou, X.-P. Zhu, J. Zhou, H.-Q. Cai, J. Tang, and Q.-L. Li, "Minocycline-released hydroxyapatite-gelatin nanocomposite and its cytocompatibility in vitro," Biomedical Materials, vol. 6, no. 2, Article ID 025002, 2011.

[12] X. Song, F. Ling, L. Ma, C. Yang, and X. Chen, "Electrospun hydroxyapatite grafted poly(l-lactide)/poly(lactic-coglycolic acid) nanofibers for guided bone regeneration membrane," Composites Science and Technology, vol. 79, pp. 8-14, 2013. 
[13] M. Kikuchi, Y. Koyama, T. Yamada et al., "Development of guided bone regeneration membrane composed of $\beta$-tricalcium phosphate and poly (L-lactide-co-glycolide-co- $\varepsilon$-caprolactone) composites," Biomaterials, vol. 25, no. 28, pp. 5979-5986, 2004.

[14] P. Karuppuswamy, J. R. Venugopal, B. Navaneethan, A. L. Laiva, and S. Ramakrishna, "Polycaprolactone nanofibers for the controlled release of tetracycline hydrochloride," Materials Letters, vol. 141, pp. 180-186, 2015.

[15] H. Maeda and T. Kasuga, "Control of silicon species released from poly(lactic acid)-polysiloxane hybrid membranes," Journal of Biomedical Materials Research Part A, vol. 85, no. 3, pp. 742-746, 2008.

[16] E.-J. Lee, D.-S. Shin, H.-E. Kim, H.-W. Kim, Y.-H. Koh, and J.-H. Jang, "Membrane of hybrid chitosan-silica xerogel for guided bone regeneration," Biomaterials, vol. 30, no. 5, pp. 743-750, 2009.

[17] L. J. Xu, L. Y. Jiang, C. D. Xiong, L. X. Jiang, and Y. Li, “Study on a novel double-layered composite membrane of $\mathrm{Mg}$-substituted nano-hydroxyapatite/ poly(L-lactide-co- $\varepsilon$-caprolactone): effect of different L-l act ide/ $\varepsilon$ - caprolactone ratios," Materials science and Engineering A: Structural Materials: Properties, Microstructure and Processing, vol. 615, pp. 361-366, 2014.

[18] C.-H. Lai, L. Zhou, Z.-L. Wang, H.-B. Lu, and Y. Gao, "Use of a collagen membrane loaded with recombinant human bone morphogenetic protein-2 with collagen-binding domain for vertical guided bone regeneration," Journal of Periodontology, vol. 84, no. 7, pp. 950-957, 2013.

[19] B. A. Al-Hazmi, K. S. Al-Hamdan, A. Al-Rasheed, N. Babay, H.-L. Wang, and K. Al-Hezaimi, "Efficacy of using PDGF and xenograft with or without collagen membrane for bone regeneration around immediate implants with induced dehiscencetype defects: a microcomputed tomographic study in dogs," Journal of Periodontology, vol. 84, no. 3, pp. 371-378, 2013. 

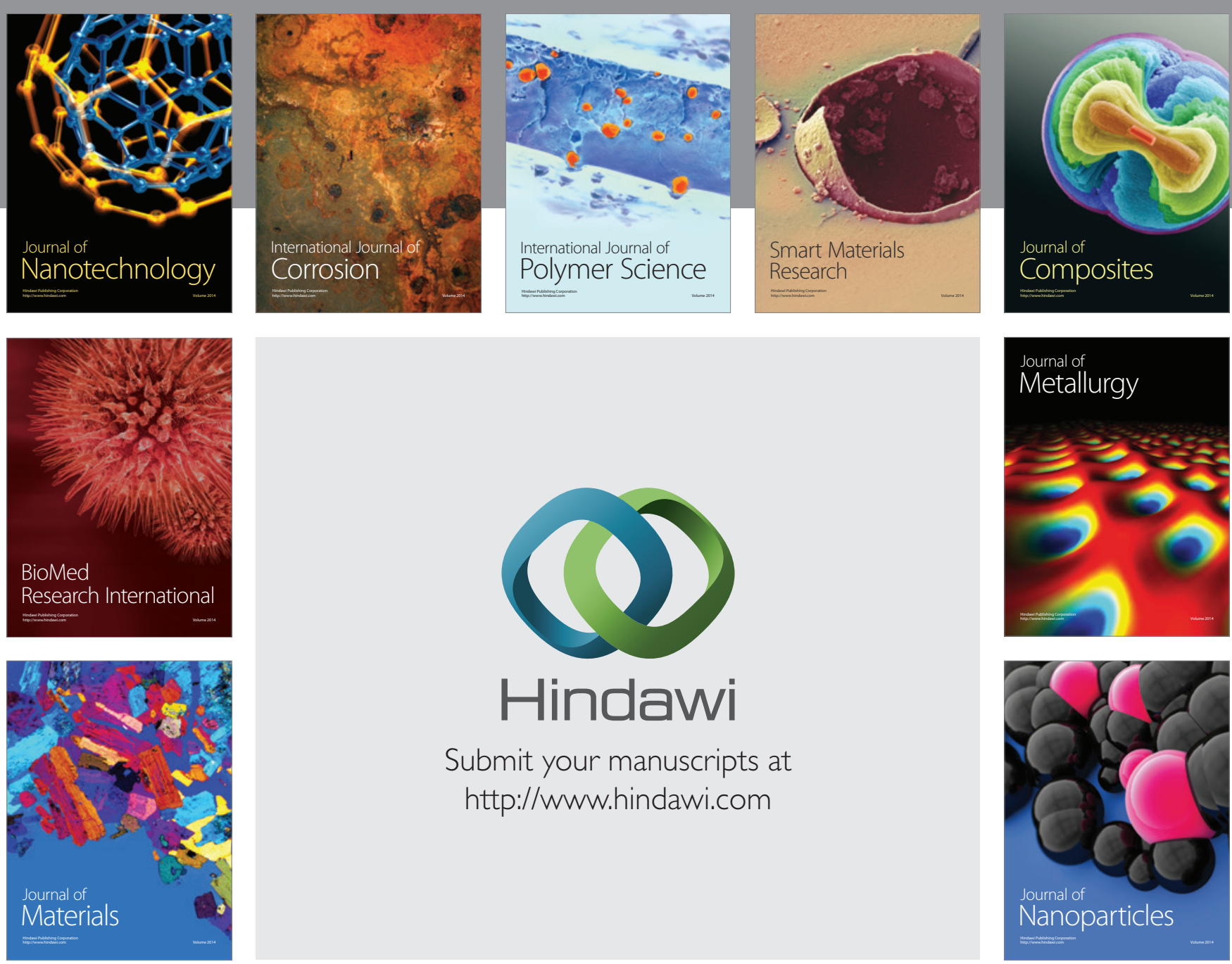

Submit your manuscripts at http://www.hindawi.com
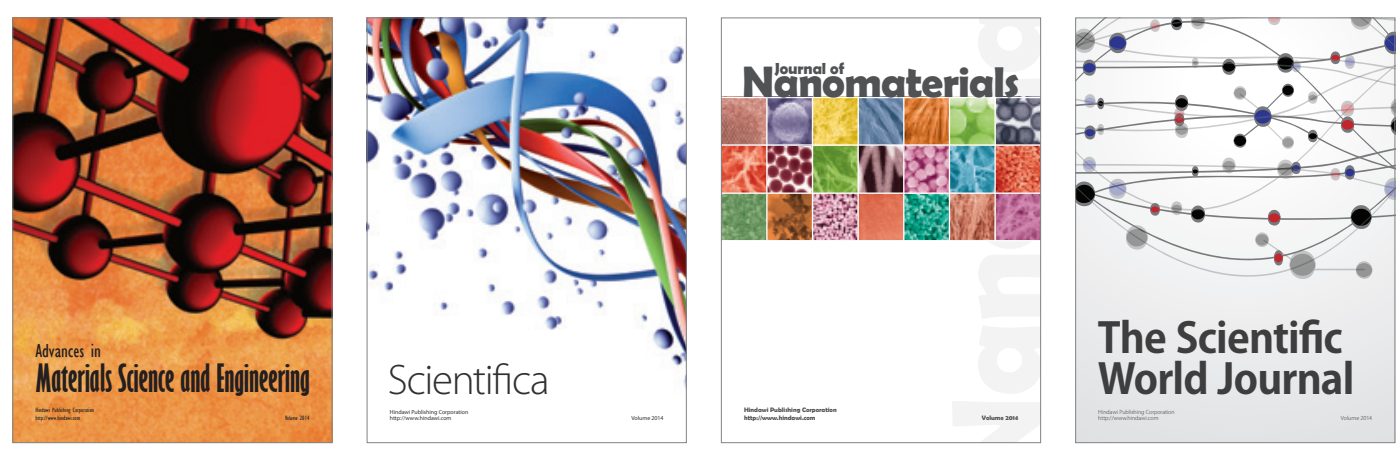

\section{The Scientific World Journal}
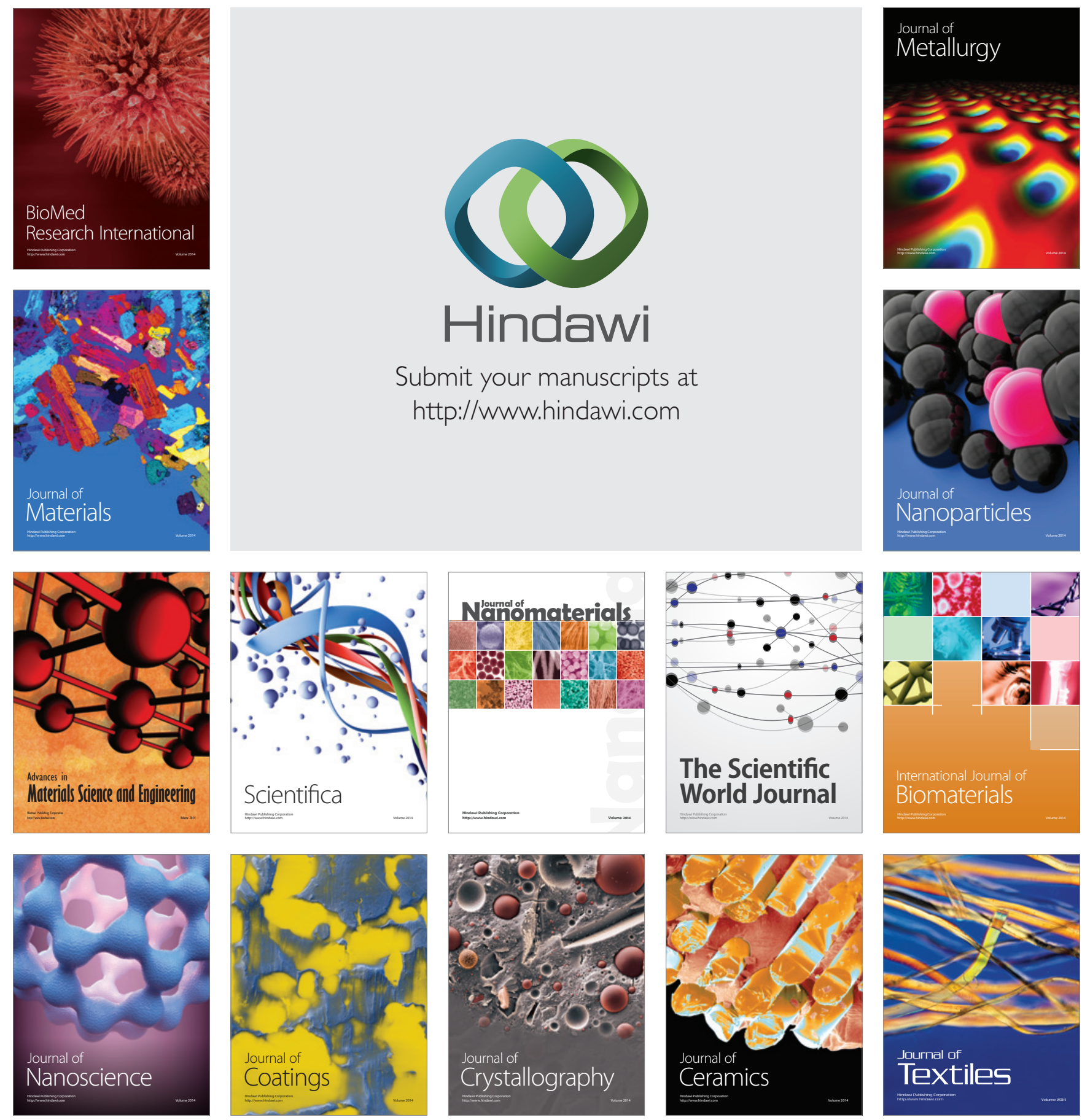